\title{
Early features of Kawasaki disease with pyuria in febrile infants younger than 6 months
}

\author{
Seo Hee Yoon, Dong Soo Kim and Jong Gyun Ahn * (D)
}

\begin{abstract}
Background: Children with Kawasaki disease (KD) and pyuria have been misdiagnosed with urinary tract infection (UTI). We compared clinical and laboratory features at admission between two groups of infants under 6 months of age who showed initial pyuria, to identify the initial clues suggestive of KD.

Methods: We retrospectively reviewed the medical records of children with fever who were under 6 months of age with pyuria, over a 10-year period (2007-2017). We included infants with sterile pyuria who were finally diagnosed with KD and those with UTI.

Results: During the period investigated, 12 (9.9\%) KD patients with sterile pyuria and 378 infants with UTI were included in this study. Older age $(P<0.01)$, a longer duration of fever; total and before admission $(P<0.01)$, more negative nitrite test $(P<0.01)$, higher platelet count $(P=0.04)$, increased $C$-reactive protein $(C R P)(P<0.01)$ and erythrocyte sedimentation rate $(E S R)(P<0.01)$, were identified as initial features of infants finally diagnosed with $\mathrm{KD}$. In the receiver operating characteristic analysis, optimal cut-off values of $509 \mathrm{k} / \mu \mathrm{L}$ for platelet count, $60 \mathrm{mg} / \mathrm{L}$ for CRP, and $68 \mathrm{~mm} / \mathrm{H}$ for ESR were selected. Patients with ESR $>68 \mathrm{~mm} / \mathrm{hr}$ had a ninefold higher odds of KD compared to those with lower ESR levels (odds ratio: 8.963, 95\% confidence intervals: 1.936-41.493, $P=0.005$ ), whereas CRP and platelet count could not significantly increase in the odds of KD at a cut-off point.
\end{abstract}

Conclusion: Persistent fever, elevated ESR, and negative urine nitrite test can serve as early clues to suspect KD in febrile infants with pyuria.

Keywords: Differential diagnosis, Fever, Infant, Kawasaki disease, Urinary tract infection

\section{Background}

Kawasaki disease (KD) is an acute systemic vasculitis of unknown aetiology that affects infants and young children [1]. KD is characterized by inflammation of all the medium-sized arteries and multisystem involvement including the kidney [2]. Renal symptoms in KD include pyuria, prerenal acute kidney injury, haemolytic uremic syndrome, acute nephritic syndrome, nephrotic syndrome, and renal tubular abnormalities [3]. Among them, pyuria is the most frequent renal feature of $K D$ occurring in $30-80 \%$ of the patients [4].

\footnotetext{
* Correspondence: JGAHN@yuhs.ac

Department of Pediatrics, Severance Children's Hospital, Yonsei University College of Medicine, 50-1 Yonsei-ro, Seodaemun-gu, Seoul 03722, South Korea
}

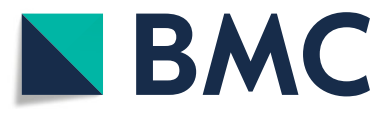

(c) The Author(s). 2018 Open Access This article is distributed under the terms of the Creative Commons Attribution 4.0 International License (http://creativecommons.org/licenses/by/4.0/), which permits unrestricted use, distribution, and reproduction in any medium, provided you give appropriate credit to the original author(s) and the source, provide a link to the Creative Commons license, and indicate if changes were made. The Creative Commons Public Domain Dedication waiver (http://creativecommons.org/publicdomain/zero/1.0/) applies to the data made available in this article, unless otherwise stated.

Some KD children with pyuria have been mistakenly attributed to urinary tract infection (UTI) [5, 6]. In particular, as infants under the age of 6 months with KD showed higher rate of incomplete presentation as well as pyuria $[2,7-9]$ that is a significant laboratory marker of UTI with high occurrence rate in these ages [10], it is a diagnostic challenge to distinguish between UTI and KD with pyuria in infants aged $<6$ month. Considering that patients with KD younger than 6 months are also known to have a higher risk of developing coronary complications $[11,12]$, it is necessary that clinicians, who evaluate febrile infant under 6 months of age with pyuria, do not miss the diagnostic potential of KD.

Therefore, it is crucial to find the initial differences among patients with UTI and KD with pyuria to identify $\mathrm{KD}$ in febrile infants with pyuria during the early phase 
of the disease. We studied the clinical and laboratory parameters of infants under 6 months of age who showed fever and pyuria at admission, in order to find initial clues for the early detection of KD that would allow timely initiation of therapy.

\section{Methods}

We retrospectively reviewed medical records of patients under 180 days of age with fever and pyuria who were admitted to Severance Children's Hospital between January 2007 and January 2017. Among these patients, we included infants who were finally diagnosed as $\mathrm{KD}$ or UTI. Diagnosis of KD was based on American Heart Association guidelines [13]. We also included incomplete $\mathrm{KD}$, which showed prolonged unexplained fever, fewer than four of the major clinical manifestations, and compatible laboratory or echocardiographic findings [2]. The definition of pyuria was based on urinary white blood cell (WBC) count $>5$ cells/high power field (HPF) and sterile pyuria is defined as pyuria with a negative urine culture $[14,15]$. The diagnosis of UTI was based on the culture of a single organism from a catheterized urine culture with a colony count greater than 10,000 or from a clean-catch midstream urine culture with greater than 100,000 colonies $[15,16]$. Two or more bacterial species isolated by culture were excluded. We only included patients with KD and with sterile pyuria in the analysis. This study was approved by the Institutional Review Board of our institution (Severance Hospital, IRB number: 4-2017-1199).

\section{Statistics}

Demographic and clinical data are presented as median (range) or frequency. Initial laboratory test data at admission were analysed. Categorical variables were compared using chi-squared test or Fisher's exact test, and continuous variables were compared by Mann-Whitney test. To compare the predictive capacity of various laboratory tests for diagnosis of $\mathrm{KD}$, receiver operating characteristic (ROC) curves were analysed by estimating the area under the curve (AUC). Optimal cut-off values in the diagnosis of $\mathrm{KD}$ were determined according to Youden index [17]. Patients were stratified into two groups according to optimal cut-off values for analyses. Logistic regression analyses were conducted using these groups (higher vs. lower than cut-off value) to estimate odds ratios and 95\% confidence intervals. Statistical analyses were performed using SPSS version 23.0 for Windows (SPSS Inc., Chicago, IL, USA) and MedCalc Statistical Software version 18.6 (MedCalc Software, Ostend, Belgium). $P<0.05$ was considered statistically significant.

\section{Results}

A total of 121 patients under 6 months of age were diagnosed with KD over the study period. Forty-one (33.9\%) of the $121 \mathrm{KD}$ children had pyuria. Of the 41 pyuria patients, 12 had sterile pyuria, 7 had concomitant UTI, and 16 had combined bacterial growth which had to be excluded. The other 6 patients were unidentified because they did not perform the urine culture tests. The clinical presentations of the $12 \mathrm{KD}$ infants with sterile pyuria are summarized in Table 1. Two patients satisfied the diagnostic criteria of complete Kawasaki disease, whereas the others were diagnosed with incomplete Kawasaki disease.

During the same period, 378 infants under 6 months of age were diagnosed with UTI. Clinical and laboratory findings between KD infants with sterile pyuria and UTI infants were compared (Table 2). Median age of KD group (5.2 months; range, 2.5-5.9 months) was older than that of UTI group (median 3.6 months; range, 0.9-6 months) $(P=0.002)$. Male-to-female ratios in KD and UTI infants were $1.4(7 / 5)$ and $2.8(277 / 101)$, respectively.

KD group showed longer duration of fever (before admission and total) compared to UTI group $(P<$ 0.01 ). KD group also showed a significantly higher platelet count $(P=0.04)$, C-reactive protein $(\mathrm{CRP})$ level $(P<0.01)$, and erythrocyte sedimentation rate (ESR) $(P<0.01)$ than UTI group.

UTI group showed higher presence of positive urine nitrite test $(P<0.01)$. There were no significant differences in white blood cell count, absolute neutrophil count, haemoglobin, aspartate aminotransferase, alanine aminotransaminase, albumin, sodium, total bilirubin, and urine $\beta 2$-microglobulin ( $\beta 2-\mathrm{MG}$ ) (Table 2).

Table 1 Clinical characteristics of patients with Kawasaki disease with sterile pyuria

\begin{tabular}{ll}
\hline Variables & $n$ (\%) or median (range) \\
\hline Typical KD/Atypical KD & $2 / 10(16.7 \% / 83.3 \%)$ \\
Female/male & $7 / 5(36.1 \% / 63.9 \%)$ \\
Age (months) & $5.2(2.5-5.9)$ \\
Total duration of fever (days) & $5.5(3-13)$ \\
Duration of fever before admission (days) & $4.5(2-9)$ \\
IVIG usage (times) & \\
1 & $11(91.7 \%)$ \\
$\geq 2$ & $1(8.3 \%)$ \\
Presence of coronary artery lesion (CAL) & $3(25 \%)$ \\
Bilateral coronary ectasia & $1(8.3 \%)$ \\
Left main artery dilatation & $1(8.3 \%)$ \\
LAD dilatation & $1(8.3 \%)$
\end{tabular}

KD Kawasaki disease, IVIG intravenous immunoglobulin, $L A D$ left anterior descending coronary artery, Data are presented as case number, percentages or median (range)

${ }^{\mathrm{a}} \mathrm{CAL}$ is defined according to the Japanese Ministry of Health and Welfare guidelines 
Table 2 Comparison between patients with Kawasaki disease and patients with urinary tract infection at admission

\begin{tabular}{|c|c|c|c|}
\hline Parameter (Median, Range) & KD with Sterile Pyuria $(n=12)$ & UTI $(n=378)$ & $P$-value \\
\hline Age (months) & $5.2(2.5-5.9)$ & $3.6(0.9-6)$ & .002 \\
\hline Male/Female & $7 / 5$ & $277 / 101$ & .321 \\
\hline \multicolumn{4}{|l|}{ Duration of fever (days) } \\
\hline Before admission & $4.5(2-9)$ & $2(1-8)$ & .000 \\
\hline Total duration & $5.5(3-13)$ & $2(1-16)$ & .000 \\
\hline WBC $(/ \mu \mathrm{L})$ & $17,620(7180-26,820)$ & $15,130(3390-33,480)$ & .232 \\
\hline ANC $(/ \mu \mathrm{L})$ & $9390(4010-15,490)$ & $8035(100-25,150)$ & .159 \\
\hline $\mathrm{Hb}(\mathrm{g} / \mathrm{dL})$ & $10.8(9.5-11.7)$ & $10.9(8.0-13.3)$ & .293 \\
\hline Platelet count $(\mathrm{k} / \mu \mathrm{L})$ & $532.5(315-772)$ & $442.0(162-1492)$ & .040 \\
\hline CRP (mg/L) & $74.6(31.6-294.5)$ & $33.7(0.3-191.9)$ & .001 \\
\hline $\mathrm{ESR}(\mathrm{mm} / \mathrm{hr})$ & $80.0(27-101)$ & $31.0(2-120)$ & .000 \\
\hline $\mathrm{Na}(\mathrm{mmol} / \mathrm{L})$ & $137(135-141)$ & $138(114-142)$ & .352 \\
\hline AST (IU/L) & $30(18-178)$ & $28(13-507)$ & .642 \\
\hline $\mathrm{ALT}(\mathrm{IU} / \mathrm{L})$ & $21(7-53)$ & $22(7-460)$ & .658 \\
\hline Albumin $(\mathrm{g} / \mathrm{dL})$ & $3.9(3.2-4.7)$ & $3.9(3.0-4.9)$ & .903 \\
\hline Total bilirubin (mg/dL) & $0.3(0.3-1.5)$ & $0.4(0.1-7.1)$ & .546 \\
\hline Urine $\beta 2-M G(m g / L)$ & $0.2(0.14-0.98)$ & $0.2(0.01-40.06)$ & .484 \\
\hline Urine nitrite test $(+)$ & $0(0 \%)$ & $201(53.2 \%)$ & .000 \\
\hline
\end{tabular}

KD Kawasaki disease, UTI urinary tract infection, WBC white blood cell, $H b$ haemoglobin, ANC absolute neutrophil count, CRP C-reactive protein, ESR erythrocyte sedimentation rate, Na sodium, AST aspartate aminotransferase, ALT alanine aminotransaminase, $\beta 2-M G, \beta 2$-microglobulin. Data are presented as case number, percentages or median (range)

We calculated the optimal cut-off values of platelet count, CRP, and ESR for differentiating KD with pyuria from UTI, by drawing ROC curves (Fig. 1). The cut-off value of platelet count to predict KD with pyuria was 509 $\mathrm{k} / \mu \mathrm{L}$ with $58.3 \%$ sensitivity, $76.5 \%$ specificity, $98.3 \%$ negative predictive value, and $7.3 \%$ positive predictive value $(P$ $=0.064)$. The cut-off value of CRP was $60 \mathrm{mg} / \mathrm{L}$ with $66.7 \%$ sensitivity, $76.1 \%$ specificity, $98.6 \%$ negative predictive value, and $8.2 \%$ positive predictive value $(P<0.001)$. The cut-off value of ESR was $68 \mathrm{~mm} / \mathrm{H}$ with $72.7 \%$ sensitivity, $85.3 \%$ specificity, $99.0 \%$ negative predictive value, and $13.8 \%$ positive predictive value $(P<0.001)$. The area under the curve (AUC) was 0.674 (95\% confidence interval $[\mathrm{CI}]: 0.625-0.720, P=0.064)$ for platelet count, 0.749 (95\% CI: 0.703-0.791, $P<0.001$ ) for CRP, and 0.846 (95\% CI: 0.804-0.882, $P<0.001)$ for ESR. Subjects with ESR > $68 \mathrm{~mm} / \mathrm{hr}$ had a nine-folds higher odds of KD compared to those with lower ESR level (odds ratio: 8.963 , 95\% confidence intervals: 1.936-41.493, $P=0.005)$ (Table 3).

\section{Discussion}

This study demonstrates that there are different initial features at admission between KD and UTI groups. Older age, longer duration of fever, higher platelet count, higher level of CRP and ESR, and more urine negative nitrite test were observed in KD group compared to UTI

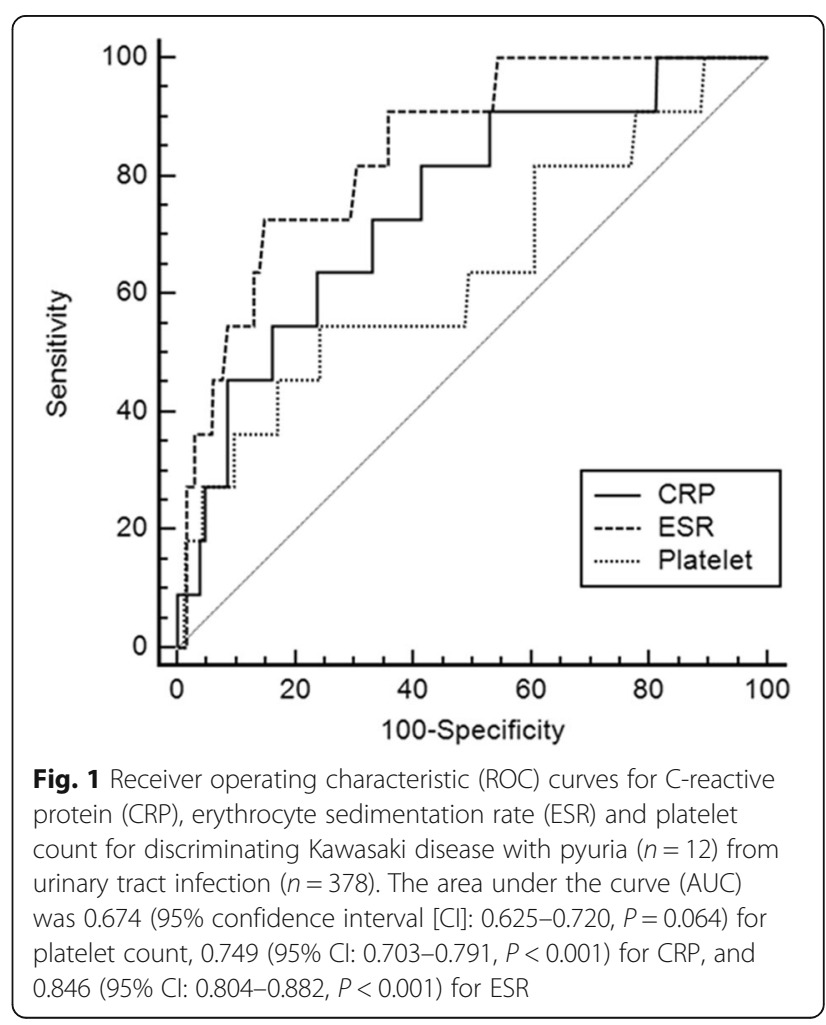


Table 3 Odds ratios for diagnosis of Kawasaki disease using cut-off levels in febrile infants with pyuria

\begin{tabular}{lll}
\hline Variables & OR $^{*}(95 \% \mathrm{Cl})$ & $P$-value \\
\hline Platelet count $(>509 \mathrm{k \mu} / \mathrm{L})$ & $2.299(0.633-8.349)$ & .206 \\
CRP $(>60 \mathrm{mg} / \mathrm{L})$ & $2.169(0.523-8.998)$ & .286 \\
ESR $(>68 \mathrm{~mm} / \mathrm{hr})$ & $8.963(1.936-41.493)$ & .005
\end{tabular}

$O R$ odds ratio, $C l$ confidence interval, $C R P$ C-reactive protein, ESR erythrocyte sedimentation rate. Odds ratio, $95 \%$ confidence interval and $P$ value were calculated by binary logistic regression analysis

*Higher vs. lower than cut off value

group. Our findings can provide early indicators for the early detection of KD in febrile infants with pyuria.

In the present study, the median age of KD group (5.2 months; range, 2.5-5.9 months) was older than that of UTI group (3.6 months; range, 0.9-6 months) $(P=$ $0.002)$. The age difference could be attributed to the fact that neonatal KD is extremely rare [18]. In a previous study, authors examined patients with KD younger than 6 months and reported that the median age was 5 months (range, 2-6) [12], or a mean age was $4.6 \pm 3.5$ months (range, 2-6) [7]. This is similar to the age range of the KD group in our results. Alternatively, the pooled prevalence rates of febrile UTI aged under 3 months ranges $7.5 \%$ (female) to $20.1 \%$ (uncircumcised males) [10]. Therefore, pyuria occurring in a febrile infant less than at least 2 months is more likely to be caused by UTI.

The diagnosis of KD is based on clinical criteria, but it could appear and progress in several days after onset of fever. Wu et al. [6] reported two cases of persistent fever and pyuria which were the initial presentation without signs suggestive of $\mathrm{KD}$, and coronary artery abnormalities were noted in both cases. In our study, KD group showed longer fever duration of total and before admission compared to UTI group. In the infants under aged 6 months, prolonged fever might be the only clinical symptom of KD [2]. Thus, early suspicion of KD should be considered in any infant with prolonged fever and culture-negative pyuria.

The CRP, ESR, and platelet count were also higher in KD group than in UTI group in this study. Elevation of acute phase reactants such as CRP and ESR is characteristic laboratory findings of KD [2]. However, they are general indicators of an acute inflammatory process and also increased in UTI, especially upper UTI [19]. Thrombocytosis, classified as a secondary thrombocytosis, is also seen in the upper UTI as well as in KD [2, 20]. Gofrit et al. [21] reported thrombocytosis in a patient with upper UTI is not a random phenomenon, but a marker of kidney obstruction or perinephric abscess. Moreover, ESR $>68 \mathrm{~mm} / \mathrm{hr}$ showed statistically significant increase in the odds of KD, whereas CRP and platelet count could not significantly increase in the odds of
KD at a cut-off point in our study. Therefore, increased ESR itself could also be a good diagnostic marker for KD. However, the laboratory markers should be interpreted cautiously and used as a supportive method to diagnose patients KD using pyuria, in consideration of nonspecificity.

In urinalysis findings except for pyuria, urine nitrite test is helpful for diagnosis of UTI for its high specificity [22]. In this study, UTI group showed higher presence of positive urine nitrite test than KD group $(P<0.01)$. Among UTI patients, $53.2 \%$ had positive nitrite test but none in the KD infant. The findings suggest that urine nitrite test could be a useful marker to exclude KD if it is positive.

Sterile pyuria in KD was thought to be due to urethritis caused by a non-specific vasculitis of the urethra and/or the kidney as a result of mild and sub-clinical renal injuries $[4,23]$. Urinary $\beta 2-\mathrm{MG}$ is being used as a useful indicator to check the function of the renal-urinary tract. In our study, urinary $\beta 2-\mathrm{MG}$ is not statistically different in KD and UTI groups. Choi et al. [24] reported that urine $\beta 2-\mathrm{MG}$ was elevated in patients with $\mathrm{KD}$ and showed no difference between KD with pyuria and without pyuria groups. This result indicates the damage on renal function in most patients with KD.

A major limitation of our study is the small population size, which may have affected our statistical results. Future analysis, with a large sample size, is needed to verify the results of this study. Despite such shortcomings, our data can still provide information regarding initial indicators of KD in febrile infants with pyuria, which will be useful for clinicians.

\section{Conclusions}

Prolonged fever duration, elevated ESR, and negative urine nitrite test can serve as early clues to suspect KD in febrile infants with pyuria. Therefore, in patients presenting with these features as well as persistent fever despite appropriate antibiotic treatment for UTI, echocardiography is warranted to identify the possible presence of KD.

\section{Abbreviations \\ AUC: Area under the curve; Cl: Confidence interval; CRP: C-reactive protein; ESR: Erythrocyte sedimentation rate; HPF: High power field; KD: Kawasaki disease; OR: Odds ratio; ROC: Receiver operating characteristic; UTI: Urinary tract infection; WBC: White blood cell; $\beta 2-M G$ : $\beta 2$-microglobulin}

Acknowledgements

Not applicable.

Funding

This study has not been funded.

Availability of data and materials

The datasets used and/or analysed during the current study are available from the corresponding author on reasonable request. 


\section{Authors' contributions}

SHY primary responsibility for data collection, preliminary data analysis and writing the manuscript. DSK participated in the study design and data evaluation. JGA supervised the design and execution of the study, performed the final data interpretation and contributed the writing of the manuscript. All authors have read and approved the final manuscript.

\section{Ethics approval and consent to participate}

This study was approved by the Institutional Review Board at Severance Hospital (approval number: 4-2017-1199). Written consent was not necessary for this study due to its retrospective nature.

\section{Consent for publication}

Not applicable.

\section{Competing interests}

The authors declare that they have no competing interests.

\section{Publisher's Note}

Springer Nature remains neutral with regard to jurisdictional claims in published maps and institutional affiliations.

Received: 28 April 2018 Accepted: 3 December 2018

Published online: 20 December 2018

\section{References}

1. Burns JC, Glode MP. Kawasaki syndrome. Lancet. 2004;364(9433):533-44

2. McCrindle BW, Rowley AH, Newburger JW, Burns JC, Bolger AF, Gewitz M, et al. Diagnosis, treatment, and long-term Management of Kawasaki Disease: a scientific statement for health professionals from the American Heart Association. Circulation. 2017;135(17):e927-e99.

3. Watanabe T. Kidney and urinary tract involvement in Kawasaki disease. Int J Pediatr. 2013;2013:831834.

4. Watanabe T. Pyuria in patients with Kawasaki disease. World J Clin Pediatr. 2015;4(2):25-9

5. Ristoska-Bojkovska N, Stavric K, Tasic V. Kawasaki disease misdiagnosed as acute pyelonephritis. Pediatr Nephrol. 2003;18(8):851-2.

6. Wu CY, Hsieh KS, Chiou YH, Wang RS, Huang IF, Lee WY, et al. Prolonged fever and pyuria: a urinary tract infection presentation of incomplete Kawasaki disease. Acta Paediatr. 2005;94(3):375-7.

7. Chang FY, Hwang B, Chen SJ, Lee PC, Meng CC, Lu JH. Characteristics of Kawasaki disease in infants younger than six months of age. Pediatr Infect Dis J. 2006:25(3):241-4

8. Yeom JS, Park JS, Seo JH, Park ES, Lim JY, Park CH, et al. Initial characteristics of Kawasaki disease with cerebrospinal fluid pleocytosis in febrile infants. Pediatr Neurol. 2012;47(4):259-62.

9. Liu HC, Lo CW, Hwang B, Lee PC. Clinical manifestations vary with different age spectrums in infants with Kawasaki disease. ScientificWorldJournal. 2012;2012:210382

10. Shaikh N, Morone NE, Bost JE, Farrell MH. Prevalence of urinary tract infection in childhood: a meta-analysis. Pediatr Infect Dis J. 2008;27(4):302-8.

11. Rosenfeld EA, Corydon KE, Shulman ST. Kawasaki disease in infants less than one year of age. J Pediatr. 1995;126(4):524-9.

12. Yoon YM, Yun HW, Kim SH. Clinical characteristics of Kawasaki disease in infants younger than six months: a single-center study. Korean Circ J. 2016; 46(4):550-5.

13. Newburger JW, Takahashi M, Gerber MA, Gewitz MH, Tani LY, Burns JC, et al. Diagnosis, treatment, and long-term management of Kawasaki disease: a statement for health professionals from the committee on rheumatic fever, endocarditis, and Kawasaki disease, council on cardiovascular disease in the young, American Heart Association. Pediatrics. 2004;114(6):1708-33.

14. Hooker JB, Mold JW, Kumar S. Sterile pyuria in patients admitted to the hospital with infections outside of the urinary tract. J Am Board Fam Med. 2014;27(1):97-103.

15. Jan SL, Wu MC, Lin MC, Fu YC, Chan SC, Lin SJ. Pyuria is not always sterile in children with Kawasaki disease. Pediatr Int. 2010;52(1):113-7.

16. Robinson JL, Finlay JC, Lang ME, Bortolussi R. Urinary tract infections in infants and children: diagnosis and management. Paediatr Child Health. 2014:19(6):315-9.

17. Youden WJ. Index for rating diagnostic tests. Cancer. 1950;3(1):32-5.
18. Hangai M, Kubota Y, Kagawa J, Yashiro M, Uehara R, Nakamura Y, et al. Neonatal Kawasaki disease: case report and data from nationwide survey in Japan. Eur J Pediatr. 2014;173(11):1533-6.

19. Ayazi P, Mahyar A, Daneshi MM, Jahani Hashemi H, Pirouzi M, Esmailzadehha N. Diagnostic accuracy of the quantitative C-reactive protein, erythrocyte sedimentation rate and white blood cell count in urinary tract infections among infants and children. Malays J Med Sci. 2013;20(5):40-6.

20. Garoufi A, Voutsioti A, Getsi VC, Zeis P, Karpathios T. Platelet changes in urinary tract infections. Pediatr Res. 1999;45:903.

21. Gofrit ON, Shapiro A, Rund D, Verstandig AG, Landau EH, Katz R, et al. Thrombocytosis accompanying urinary tract infection suggests obstruction or abscess. Am J Emerg Med. 2006;24(1):118-21.

22. Roberts KB. Urinary tract infection: clinical practice guideline for the diagnosis and management of the initial UTI in febrile infants and children 2 to 24 months. Pediatrics. 2011;128(3):595-610.

23. Watanabe T, Abe Y, Sato S, Uehara Y, Ikeno K, Abe T. Sterile pyuria in patients with Kawasaki disease originates from both the urethra and the kidney. Pediatr Nephrol. 2007;22(7):987-91.

24. Choi JY, Park SY, Choi KH, Park YH, Lee YH. Clinical characteristics of Kawasaki disease with sterile pyuria. Korean J Pediatr. 2013:56(1):13-8.

\section{Ready to submit your research? Choose BMC and benefit from:}

- fast, convenient online submission

- thorough peer review by experienced researchers in your field

- rapid publication on acceptance

- support for research data, including large and complex data types

- gold Open Access which fosters wider collaboration and increased citations

- maximum visibility for your research: over $100 \mathrm{M}$ website views per year

At BMC, research is always in progress.

Learn more biomedcentral.com/submissions 